\title{
CONTENT BASEd DATA TRANSFER MECHANISM FOR EFFICIENT BULK DATA TRANSFER IN GRID COMPUTING ENVIRONMENT
}

\author{
N. Thenmozhi ${ }^{1}$ and Dr. M. Madheswaran ${ }^{2}$ \\ ${ }^{1}$ N.K.R. Govt. Arts College for Women, Namakkal, India, 637001. \\ nthenmozhi@yahoo.co.in \\ ${ }^{2}$ Muthayammal Engineering College, Rasipuram, India, 637408. \\ madheswaran.dr@gmail.com
}

\begin{abstract}
A new class of Data Grid infrastructure is needed to support management, transport, distributed access, and analysis of terabyte and peta byte of data collections by thousands of users. Even though some of the existing data management systems (DMS) of Grid computing infrastructures provides methodologies to handle bulk data transfer. These technologies are not usable in addressing some kind of simultaneous data access requirements. Often, in most of the scientific computing environments, a common data will be needed to access from different locations. Further, most of such computing entities will wait for a common scientific data (such as a data belonging to an astronomical phenomenon) which will be published only when it is available. These kinds of data access needs were not yet addressed in the design of data component Grid Access to Secondary Storage (GASS) or GridFTP. In this paper, we address an application layer content based data transfer scheme for grid computing environments. By using the proposed scheme in a grid computing environment, we can simultaneously move bulk data in an efficient way using simple subscribe and publish mechanism.
\end{abstract}

\section{KEYWORDS}

GASS, GridFTP, Content Based Routing, Bulk Data Transfer, ERNET, GARUDA.

\section{INTRODUCTION}

Grid computing can be used in a variety of ways to address various kinds of application requirements. Based on the structure of the organization, principle resources and on the basis of their use it is categorized into three primary types which are summarized below. Of course, there are no hard boundaries between these grid types [1].

Computational grid: A computational grid is a hardware and software infrastructure that provides dependable, consistent, pervasive and inexpensive access to high end computational capabilities.

Scavenging grid: A scavenging grid is most commonly used with large numbers of desktop machines. Machines are scavenged for available CPU cycles and other resources. Owners of the desktop machines are usually given control over it when their resources are available to participate in the grid.

Data grid: A data grid is responsible for housing and providing access to data across multiple organizations. Users are not concerned with where this data is located as long as they have access 
to the data. For example, you may have two universities doing life science research, each with unique data. A data grid would allow them to share their data, manage the data, and manage security issues such as who has to access what data.

Content-based communication is a communication service whereby the flow of messages from senders to receivers is driven by the content of the messages, rather than by explicit address assigned by senders and attached to the messages [3]. Using a content-based communication service, receivers declare their interests by means of selection predicates, while senders simply publish messages. The service consists of delivering to any and all receivers, each message that matches the selection predicates declared by those receivers.

Content-based communications is ideally suited for a variety of application domains, including news distribution, publish and subscribe event notification, system monitoring and management, network intrusion detection, service discovery, data sharing, distributed electronic auctions, and distributed games. A content-based network is a point-to-point, application level overlay consisting of client nodes and router nodes, connected by communication links. A content-based network can be thought of as a dynamically configurable broadcast network, where each message is treated as a broadcast message whose broadcast tree is dynamically pruned using content-based addresses.

In this paper, we study the usability of content based data transfer mechanism for grid computing environment.

\section{RELATED WORKS}

The Storage Resource Broker (SRB) [4] is middleware infrastructure that provides a uniform, UNIX-style file I/O interface for accessing heterogeneous storage resources distributed over wide area networks. Using its Metadata Catalog (MCAT), SRB provides collection-based access to data based on high-level attributes rather than on physical filenames. SRB also supports automatic replication of files on storage systems controlled by SRB. In contrast to the layered Globus architecture with direct user and application control over replication, SRB uses an integrated architecture, with all access of data via the SRB interface and MCAT and with SRB control over replication and replica selection.

The Active Data Repository [6] and Data Cutter [5] systems support analysis of large datasets, and could be used to achieve high-performance operations. Antonio Carzaniga et al [3] used traditional broadcast protocol combined with a specific content based protocol and used a push pull mechanism for the propagation of routing information, he evaluated the protocol delivery messages to nodes, prevent unnecessary message traffic and produce a reasonable and stable amount of control traffic.

Hiroyuki Ohsaki and Makoto Imase [12] discussed that GridFTP is a data transfer protocol in grid computing which is widely used for transferring a large volume of data efficiently. Wen-Zhan Song and Xiang-Yang Li [13] proposed an innovative peer-to-peer system architecture called CBRBrain to implement the content based routing services over the backbone routers instead of at the terminal hosts to improve the efficiency and security.

The author Nader Mohameda et al [14] used Java Object Router (JOR), it is an application level router that eliminates the limitation of contents based routers employ XML extensible content based object router that goes beyond message to routing entire java objects. Also XML limits the object sent to 3 types of data, document and messages. Geoff Coulson et al [15] gives Gridkit approach which integrates middleware and overlay network functionality and eliminating exiting grid middleware is poorly equipped to support next generation grid applications. 
International Journal of Grid Computing \& Applications (IJGCA) Vol.2, No.4, December 2011

Piotr Grzybowski's [16] Data Manament System (DMS) main task is to store and manage computational data. Sasu Tarkoma examined the cost of publisher and subscriber mobility using 3 mobility mechanisms and topologies such as generic mobility support, acyclic graphs and rendezvous based topologies. He also discussed the impact of completeness and incompleteness of the publisher and subscriber topology on the cost of mobility and identified generic mechanism has a high cost for mobility, and the other two are considerably at lower cost [17].

In a previous work [19] Stefano Castelli, Paolo Costa observed that it is able to tolerate high churn with low overhead by evaluating the author proposed HYPERCBR approach in two relevant CBR contents, such as content based searches in P2P networks and content based publish and subscribe. Roberto Baldoni et al [20] explored a new approach to content based routing in mobile Ad Hoc Networks. In it messages are broadcast efficiently to all neighbouring nodes and differs the nodes in their decision to forward the message based on an estimation of their distance from potential subscriber of the message. The simulation shows that the messages can be delivered with high probability to the interested subscribers at a low cost.

Phillip M. Dickens, and Illino [21] discussed about a high performance file transfer mechanism for grid computing to achieve excellent performance in a computational grid and he used the concept of Lightweight Object-Based (LOB) file transfer System. He shown that it is able to achieve data transfer rates of $35 \mathrm{MB}$ per second on one set of endpoints connected by the Abilene backbone network.

Hari Subramoni et al [24] designed a Advanced Data Transfer Service (ADTS), to provide the low-level InfiniBand which supports to the Globus X10 layer, and introduced the concept of I/O staging in Globus X10 ADTS driver (designed based on TCP/IP, UDP) to achieve efficient disk based data transfer in both LAN and WAN.

Wantao Liu et al [25] framework design were meet the data transfer requirements to move large number of relatively small files reliably and rapidly to remote locations and building on GridFTP the system uses combination of automatic concurrency adaptation and restart mechanisms to move large volumes of data with high performance. Also his scheduling policies support the specification of dependencies between transfers and multiple network paths. Their results indicate data transfer system can significantly improve data transfer performance and can recover well failures.

The request manager depends in particular on two components of the Globus Toolkit: the replica catalog for replica location and GridFTP for secure, efficient transfer. In addition, it uses Message Digest (MDS)-2 for access to Network Weather Service (NWS information), and Grid Security Infrastructure for authentication. This uses of Globus Toolkit components is consistent with proposals for a data grid architecture [7, 8] which includes four levels: fabric, connectivity, resource and collective.

At the lowest fabric level of the architecture is the basic components that make up the Grid, including storage systems, networks and computational systems. In addition, it includes two catalogs: a metadata catalog and a replica catalogs are where registered replica's information is stored. At the connectivity layer there are various standard protocols for communication and security. At the resource level there are services associated with managing individual resources GridFTP is placed here, which is used for the MDS information service and the Grid Resource Allocation. Management (GRAM) protocol is used for resource management.

Finally, at the collective layer there is higher level services that manages multiple underlying resources, including services provided by the request manager such as reliable file transfer, replica selection and information services that provide resource discovery or performance 
estimation. GridFTP and a replica catalog Application Programming Interface (API) services are used to construct a range of higher-level data management services. Such as reliable creation of a copy of a large data collection at a new location and selection of the best replica for a data transfer based on performance estimates provided by information services $[9,10]$.

The GridFTP data transfer protocol developed by the Globus project that provides efficient, reliable, secure data transfer in grid computing environments. The request manager initiates and monitors GridFTP data transfers.

GridFTP was developed as a protocol that aims at effective transfer of large amount of data in Grid computing. GridFTP is extending as FTP by circumventing the problems with TCP. In particular, GridFTP supports a feature to negotiate the size of the TCP socket buffer (i.e., automatic negotiation of the TCP socket buffer size) as well as a feature to use multiple TCP connections to transfer a single file in parallel (i.e., parallel data transfer). Because of these features, GridFTP has proved to be more effective than traditional FTP in transferring data especially over a network with large bandwidth-delay product [12].

In a data grid environment RM supports the management, distributed access, huge data sets by thousands of researchers. Management of replicated data is an important function. The Globus data grid toolkit is used in this work with provided layered replica management architecture. At the lowest level, a Replica Catalog allows users to register files as logical collections and provides mappings between logical names for files and collections and the storage system locations of file replicas. The catalog registers three types of entries: logical collections, locations, and logical files. Building on this basic component, a low-level API performs catalog operations. This API can be used by higher-level tools such as the request manager that select among replicas based on network or storage system performance [2].

Globus Toolkit contains a data management component that provides efficient method for moving files and data to various nodes with in the grid environment. This component, know as Grid Access to Secondary Storage (GASS). It includes facilities such as GridFTP and utilizes the GSI for user authentication and authorization. Therefore, once a user has an authenticated proxy certificate, he can use the GridFTP facility to move files without having to go through a login process to every node involved [1]. This facility provides third-party file transfer so that one node can initiate a file transfer between two other nodes.

GridFTP is designed to provide a superset of the features offered by the various Grid storage systems currently in use. But, often, more than one entity in a grid computing network will be waiting for a common data which will be published only after some time. In such cases, on the arrival of such new data, lot of redundant data transfer or duplicate flows will be in the network. This kind of redundant data transfer will consume lot of network resources. So for such scenario, we propose a content based data transfer model for efficient data transfer in grid network. We will study the performance of the proposed data transfer model in the Indian Grid Network Topology called GARUDA.

ERNET[26] (Education and Research Network) was the first dedicated and integrated step towards enabling the research and education community in India to leverage the benefits of Information and Communication Technologies. ERNET India aims at developing, setting up and operating nationwide state-of-the-art computer communication infrastructure and providing services to the users in academic and research institutions, Government organizations, and industry, in line with technology developments and national priorities. Dissemi nation, training and knowledge transfer in the field of computer communication and information technology are an integrating part of ERNET mission. 
International Journal of Grid Computing \& Applications (IJGCA) Vol.2, No.4, December 2011

The network comprises a mix of terrestrial and satellite-based wide area networks. It provides a wide range of operation and application services. As of today, universities, academic institutions, R\&D labs and schools, etc. use ERNET for a variety of applications and services including email, file transfer, database access, world wide web , web hosting, mail relaying, security solutions, distant learning and grids.

ERNET is the first network in the country to provide dual stack access of Internet protocol version 6 (IPv6) and Internet protocol version 4 (IPv4) test beds to its users to develop, test and implement IPv6 based mail, Domain name Services, Web applications and products.

ERNET has deployed many overlay networks over its terrestrial and satellite network under different schemes. Some examples are GARUDA, University Grant Commission (UGC)-Infonet, interconnecting Indian universities, Indian Council for Agriculture Research-Net (ICAR-Net), interconnecting Agricultural Research centers, Universities and Stations, and several pilot projects aiming at interconnecting schools. Separate networks were implemented to allow Department of Automic Energy (DAE) institutes to connect to the GÉANT network and to participate in Large Hadron Collider(LHC) activities.

GARUDA[27] initiative is a collaboration of science researchers and experimenters on a nationwide grid of computational nodes, mass storage and scientific instruments that aims to provide the technological advances required to enable data and compute intensive science of the 21st century. One of GARUDA's most important challenges is to strike the right balance between research and the daunting task of deploying that innovation into some of the most complex scientific and engineering endeavours being undertaken today.

The Department of Information Technology (DIT) has funded the Center for Development of Advanced Computing (C-DAC])[30] to deploy the nationwide computational grid 'GARUDA' which today connects 45 institutions across 17 cities in its Proof of Concept (PoC) phase with an aim to bring "Grid" networked computing to research labs and industry. In pursuit of scientific and technological excellence, GARUDA PoC has also brought together the critical mass of well established researchers.

The GARUDA network is a Layer 2/3 MPLS Virtual Private Network [VPN] connecting selected institutions at 10/100 Mbps with stringent quality and Service Level Agreements. The network has been contracted as a managed service with a peak capacity of $2.43 \mathrm{Gbps}$ across 17 cities. This network is a precursor to the next generation Gigabit speed nationwide Wide Area Network with high performance computing resources and scientific instruments for seamless collaborative research and experiments[29][30]. The PoC network was established at all the GARUDA partner institutes in close collaboration with ERNET who are responsible for the operation, maintenance and management of this network.

\section{MODELING GRID AND CONTENT BASED DATA TRAFFIC FOR GRID IN NETWORK SIMULATOR NS2}

The Grid Computing paradigm has been widely adopted within the research community for scientific computing. Grid Computing is used as a method by which access is seamlessly given to a set of heterogeneous computational resources across a dynamic set of physical organizations, supplying massive computing and storage capabilities. Within a Grid environment, computational jobs are submitted to and run on suitable resources. The data is stored and transferred transparently without knowing its geographic location. All of this behaviour will obviously have impact on the underling network infrastructure and the data generated within a Grid environment may substantially affect the network performance due to the volume involved. NS2 simulator is used to simulate the network. 
International Journal of Grid Computing \& Applications (IJGCA) Vol.2, No.4, December 2011

Content-based networking is a networking concept, where no explicit addressing scheme is used to identify senders and receivers. There is a particular interest in this concept because the overhead is minimal and there is no need for the packets or data to be fetched from source all the time. The required data is got from the network as and when the content is published. A node is called publisher if it pumps data into the network. Such nodes are also called as content generators. Subscribers are nodes that are final recipients of the data. They are called as content consumers. Node in the network can be a content generator or a consumer or both or intermediate data forwarding node.

In content based networking each node whether publisher or subscriber sends a tag which is nothing but the content which the node subscribes or publishes. This is stored in a subscription list on each intermediate forwarding node. The intermediate node's tags the data generators with the content tag. Whenever the data is received from any of the data generators, the router checks the content tag and forwards the packet/data to appropriate path. This process continues and all intended recipients receive the data.

Many event systems use the operators of Boolean logic AND, OR and NOT to construct filters. The main entities are publishers (Pub) and subscribers (Sub) of information. A publisher publishes an event or message or notification (n) and a subscriber receives notifications of events that have been occurred. Event router is a component that connects the publishers and subscribers through many intermediate nodes. It consists of a set of connections to neighbouring routers and a set of local clients. Both sets are associated with a routing table that contains information about which event messages should be forwarded to which neighbouring router or local client.

Algorithm 1: Filter processing in Content Based Data Transfer.

1. The procedure will be terminating when a filter $f$ is found for which $f$ has more or equal subscription to the subscription filter $\mathrm{F}$ and $\mathrm{A}$ is a subscriber of $\mathrm{F}$. Because covering filter already subscribed filter $\mathrm{f}$ for $\mathrm{A}$.

2. The server removes A from all subscriptions covered by $F$ if a filter $f$ is found for which $\mathrm{f}$ is identical to $\mathrm{F}$ and $\mathrm{A}$ is not a subscriber of $\mathrm{f}$ and then $\mathrm{A}$ is added to the subscribes of F.

3. Otherwise, the filter $\mathrm{F}$ is placed in between two possible empty sets such as immediate predecessors and immediate successors of $\mathrm{F}$. The filter $\mathrm{F}$ is inserted and $\mathrm{A}$ is added to subscribers (F). The server removes A from all subscriptions covered by $F$.

4. In all the cases subscriptions with no subscribers are removed.

The algorithm used the following infrastructure interface operations.

Subscribes (A, F)

Publishes (A, n)

Notify $(A, n)$

Unsubscribe (A, F)

Advertises (A, F)

Unadvertises (A, F)
: A subscribes filter $\mathrm{F}$

: A publishes notification $\mathrm{n}$

: A is notified about notification $n$

: A unsubscribe filter $\mathrm{F}$

: A advertises filter $\mathrm{F}$

: A unadvertises filter $\mathrm{F}$

The proposed Content Based Data Transfer (CBDT) in Grid:

1. A publisher Node in a grid network sends out a message that it is going to transmit into content type for example an astronomical data. 
International Journal of Grid Computing \& Applications (IJGCA) Vol.2, No.4, December 2011

2. This is known as an event and this is propagated throughout the grid network.

3. Some remote client Nodes or applications in the grid network may like to receive this content.

4. Node that wants to receive sends a subscription message. Each intermediate Node in the grid network builds its portion of the routing tree, specific to the publisher.

5. When a data packet is received by an intermediate node, it compares the metadata, and then this packet is propagated through the network, based on the downstream subscriptions.

6. On the event of the data publication, the data will be routed to all the subscribed nodes of the grid network the intermediate routers which will have those subscription information in their tables.

The following block of pseudo code explains the above steps in detail. This algorithm will be implemented in each and every node of the grid network. The same copy of this agent code will run in the Publisher, Subscriber and the routing Nodes. On receiving a message, it will be treated according to the type of the received message and the type of the node receiving it.

Algorithm 2: The Pseudo Code of the Algorithm on Receiving Data(D)

1. Resolve message_id, MessageType, PublisherAddress, PublicationID

2. if (MessageType $==$ "ToBePublished") \{

2. a. if (NodeType $=="$ Router")

Update its PublicationIDsList and forward the message

2. b. if (NodeType $=="$ Subscriber ")

The subscriber knows about this future publication and may or may not

subscribe to it in future using this PublicationID

2. c. if (NodeType $==$ "PublisherNode")

Another Publication node does nothing

\}

3. if (MessageType $==$ "Publish") \{

3. a. if ( NodeType $=="$ Router") \{

for each Subscriber of this PublicationID

do \{

if (Source ! = Subscriber)

SendDataTo Subscribe

\}

\}

3. b. if (NodeType $=="$ Subscriber ")

Finally Receive the Data

3. c. if (NodeType $=="$ "PublisherNode")

do nothing

\}

4. if (MessageType $==$ "Subscribe")

4. a. if (\$NodeType $=="$ Router")

Update its CBDT routing tables And then Forward the Message

4. b. if (NodeType $=="$ Subscriber ")

Update PublishedMessagesList

4.c. if (NodeType $=="$ "Publisher") 


\section{SIMULATION RESULTS AND DISCUSSIONS}

A simple model of GARUDA grid network has been simulated in ns2 [26] and the normal data transfer as well as the proposed data transfer methods were evaluated. A normal $2 \mathrm{GHz}$ Pentium IV computer with 1 GB RAM was used for this simulation.

\subsection{The Simulation of GARUDA Network in ns2}

The following NAM (network animator) output shows the model of GARUDA network simulated on ns2. The topology was derived from the information provided by the ERNET and GARUDA projects [28][29].

The links shown in green are 8/34Mbps links

$>$ The links shown in red are $2 / 8$ Mbps links

$>$ Nodes shown as red hexagon are backbones and POPs

$>$ Nodes shown as blue circles are the connected institutes

Table 1. Simulation Parameters

\begin{tabular}{|l|l|}
\hline Simulation Parameters & Value \\
\hline Number of Simulated Institution Nodes & 36 \\
\hline Routing Protocol & DV \\
\hline Backbone Link Capacity & $8 / 34 \mathrm{Mbps}$ \\
\hline Number of Backbone and POP nodes & 12 \\
\hline Institution to Backbone Links & $2 / 8 \mathrm{Mbps}$ \\
\hline Queue Type & Drop Tail \\
\hline Number of Simulated Publishers & 2 \\
\hline Number of Simulated Subscribers & 12 \\
\hline
\end{tabular}

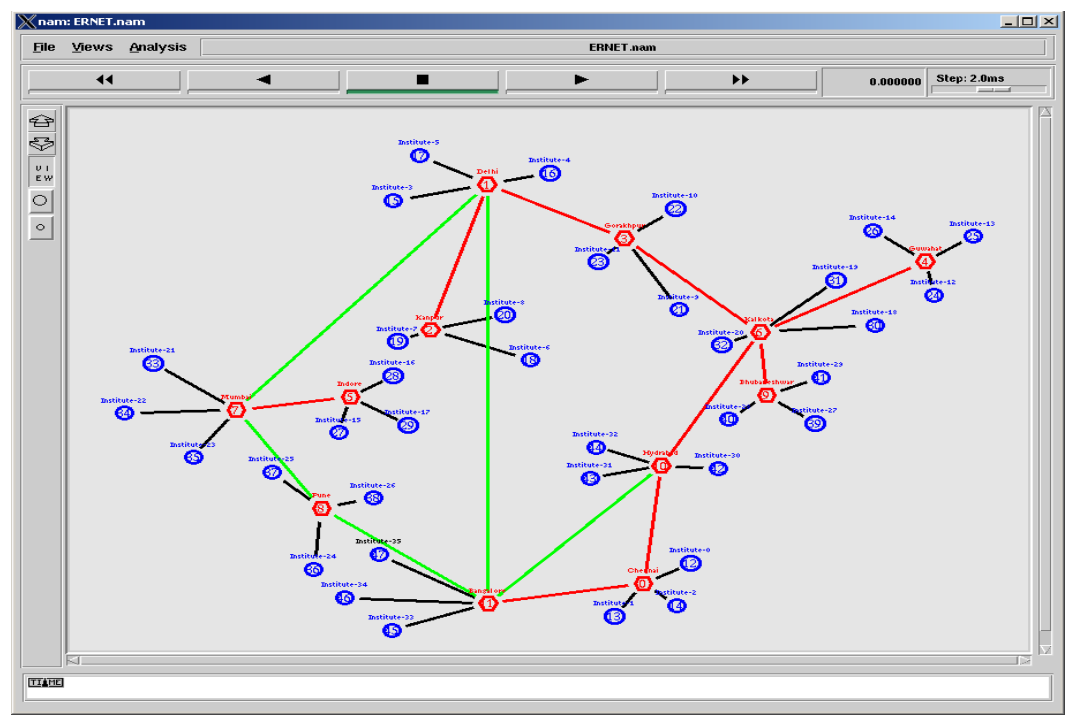

Figure 1. The Simulated GARUDA Topology[26] 
International Journal of Grid Computing \& Applications (IJGCA) Vol.2, No.4, December 2011

\subsection{The Data Traffic}

During simulating normal network, two kinds of data flows (with different packet size) of normal data transfer were used with constant bit rate traffic. Since there were 12 subscriber nodes, in normal case, there were 12 individual flows in the network. (For each kind of flow six subscribers used).

But in the proposed content based scenario, for the same kind of traffic only two major flows in the network is used, even for reaching 12 different destinations.

In this experiment, node 33 to 44 was selected as subscriber nodes. Among the 12 nodes first 6 nodes will subscribe for data stream 1 and another 6 nodes will subscribe for data stream 2. Node 26 and 27 are the publishers. Node 26 is a node which is connected in the router Guwahat and node 27 is a node which is connected through the router Indore. The node 26 (DataStream 1) and the node 27 (DataStream 2) are schedule send stream for the subscribers node 33 to 44 . If the receiving node is a publisher, it may simply forward the message or make use of it. If the receiving node is a router, it will update its CBDT routing table.

\subsection{Performance}

The following graph shows the performance of the proposed data transfer protocol with respect to the normal data transfer mechanism. The simulation runs for 50 seconds and the data were logged in the trace files and the trace files were analyzed using suitable trace analysis scripts.

As shown in the graph, the proposed data transfer scheme was able to send little bit higher than the normal scheme because, there was not that many duplicate flows or traffic in the proposed scheme.

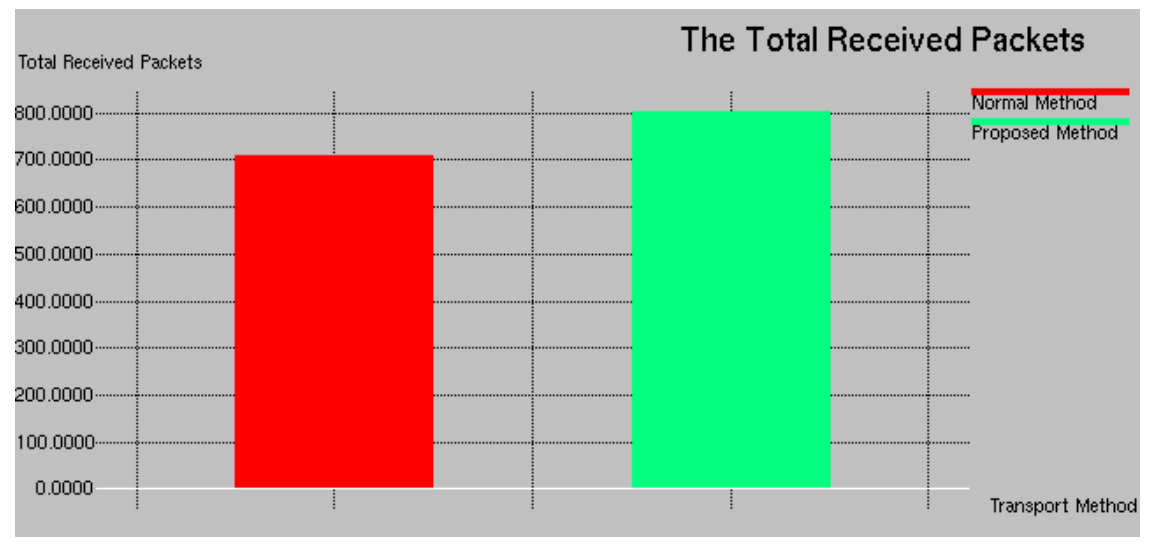

Figure 1. Total Received Data Packets

In both cases, the numbers of generated or published data packets are numerically equal. But, in the proposed case, the packets generated are very much lower than the normal scheme. Because, in normal data transfer scheme, there will be 12 individual flows during publishing the two types of data (6 flows for each type of data). This will create lot of redundancy in the network. The following figure 3 shows this obvious overhead in normal method.

Even though there will be much effort needed to build the initial subscription list in each intermediate node during the process of subscription. It will be a onetime operation. So after the completion of subscription, the data will be forwarded in an efficient way without any redundancy in the flows. So obviously the network load is very low in the proposed data transfer scheme. The figure 4 shows the difference in network load. 


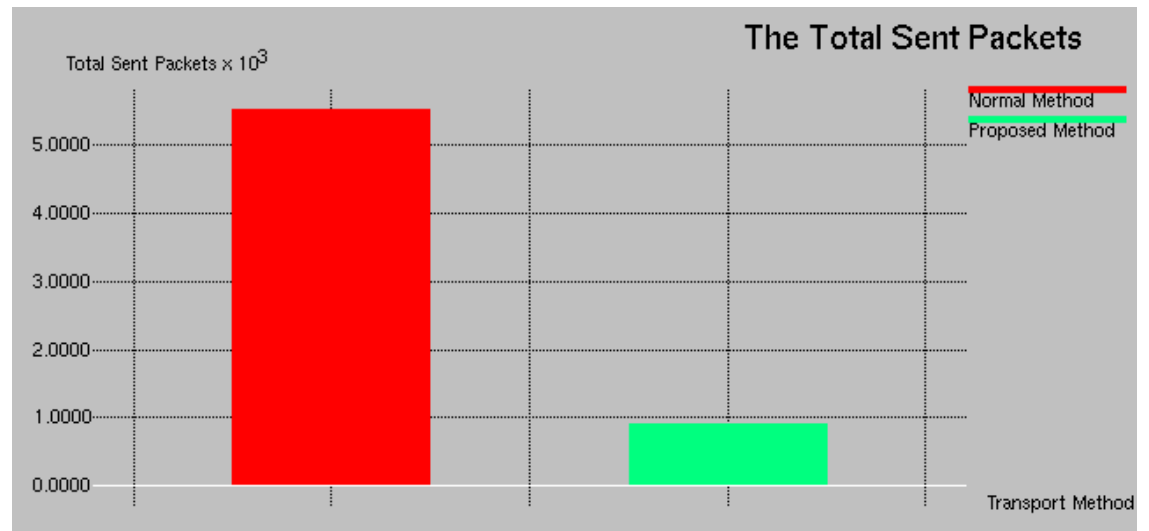

Figure 2. Total Sent Data Packets

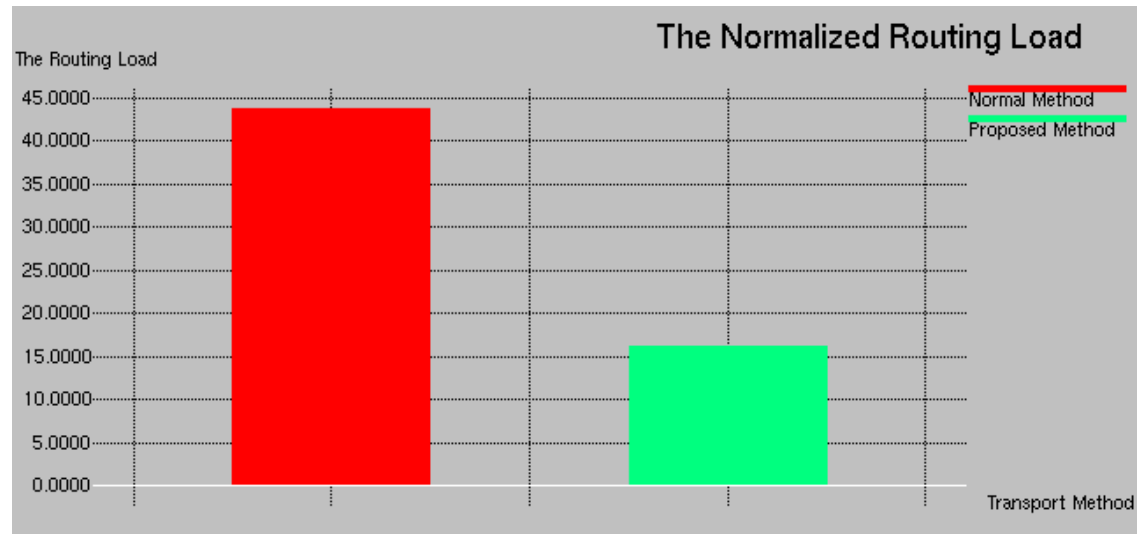

Figure 3. The Normalized Routing Load

The figure 5 shows the difference in the total data packets enqueued in intermediate nodes of the network. Since the proposed method reduces lot of duplicate packets, it is having minimum data packets to enqueue than that of the normal method. So obviously the proposed will keep the queues in the routers free and hence lead to better performance.

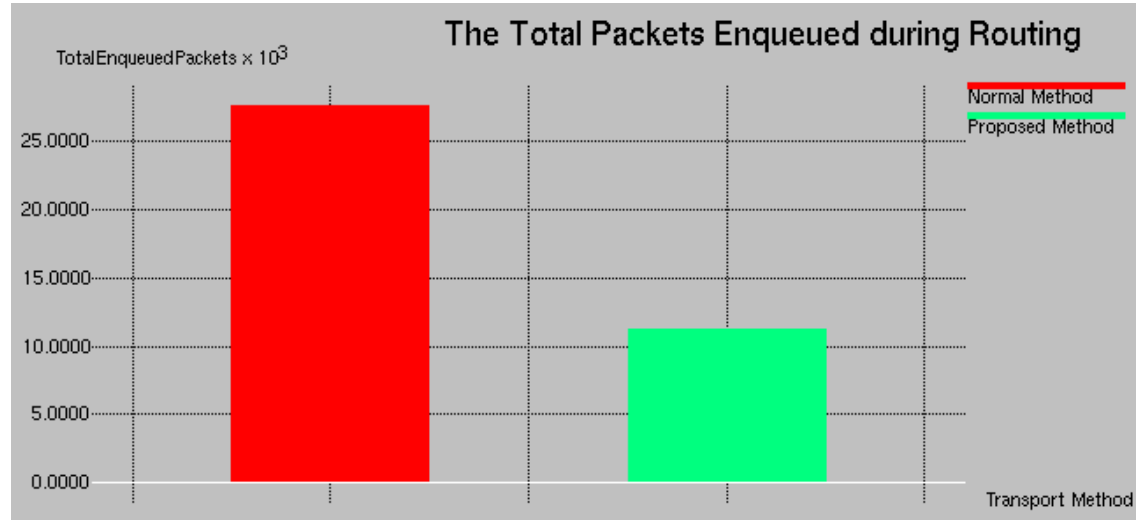

Figure 5. Total Packets Enqueued during Routing 
The following figure 6 shows the difference in the total data packets dequeued in intermediate nodes of the network. It is almost equal to that of the previous graph, because, the previously enqueued data will get dequeued for routing. It is equal because, in this experiment, we did not overload the network so that there will not be noticeable packet drop. We did these measurements in ideal traffic condition because, we want to measure the numerical difference between the normal and proposed method very clearly. So, if we introduce much cross traffic, then these bars will be little bit shorter than the previous one (due to packet drop). Since the proposed method reduces lot of duplicate packets, it is having minimum data packets to dequeue than that of the normal method. So obviously the proposed will keep the queues in the routers free and hence lead to better performance.

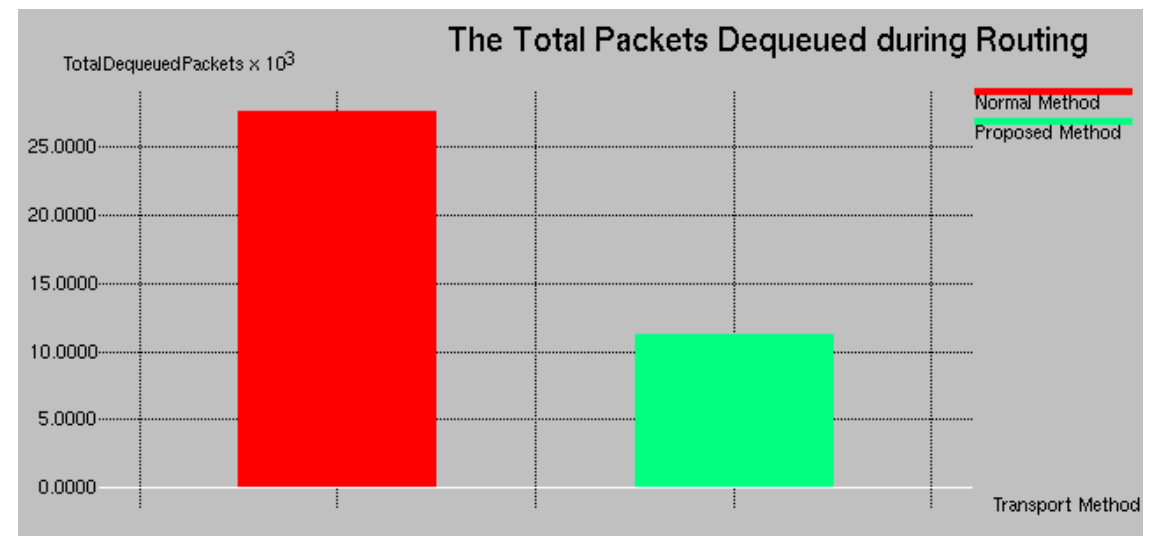

Figure 6. Total Packets Dequeued During Routing

Packet Delivery Ratio (PDF) or Packet Delivery Rate (PDR) is a ratio between total sent and received packets (It has no units). The PDF/PDR is calculated as follows:

$$
\text { PDF }=\text { Total Received Data Packets / Total Sent Data Packets } \quad x \quad 100
$$

Generally, in normal data transfer scenario, the PDF will be 100 only in ideal condition. If all the sent data packets were successfully received, then it will lead to the PDF of 100.

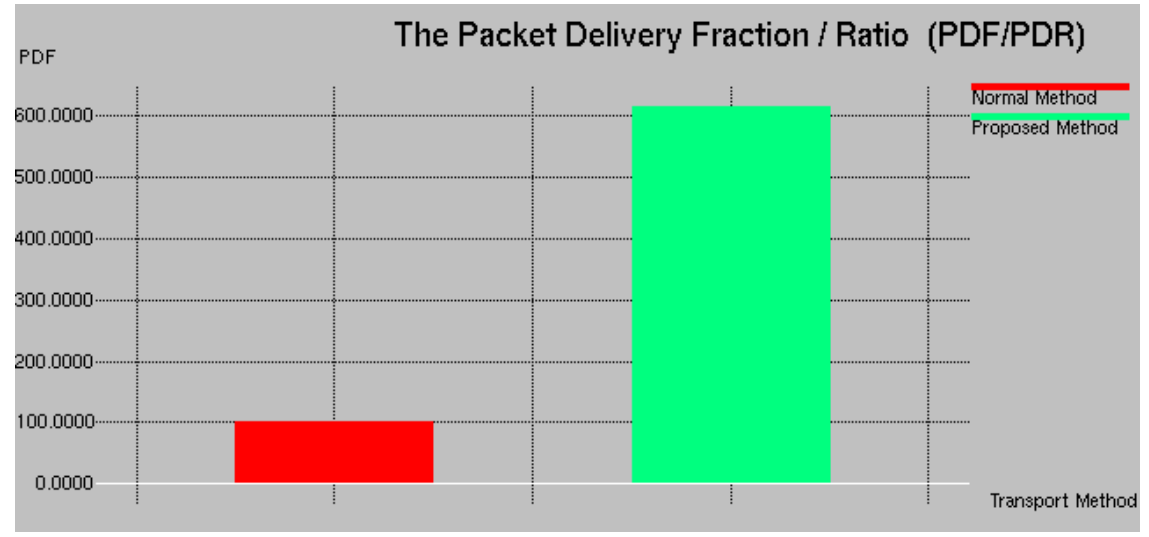

Figure 7. Total Packets Delivery Ratio (PDF)

In the normal routing protocol, if there will not be much cross traffic, then it will lead to a PDF of 100. So for normal method, we are getting the same in our simulation. But, the PDF in the case of proposed method is 600 . This is because, for each one sent data packets there may be more 
than one subscribing nodes which will receive a copy of the sent packet. So it is giving 100 as PDF in the proposed method. So it is obviously proves that the proposed method is much better than the normal method.

The following chart shows the performance of the proposed data transfer protocol with respect to the normal data transfer mechanism. The simulation runs for 50 seconds and the data were logged in the trace files and the trace files were analyzed using suitable trace analysis scripts.

Table 2. Comparison of Normal and Proposed Scheme

\begin{tabular}{|l|r|r|}
\hline \multicolumn{1}{|c|}{ Outputs/Schemes } & Normal Method & \multicolumn{1}{c|}{ Proposed Method } \\
\hline The normalized Routing Load & 43.83 & 16.13 \\
\hline The Total Received Packets & 708.00 & 804.00 \\
\hline The Total Sent Packets & 5520.00 & 919.00 \\
\hline $\begin{array}{l}\text { The Total Packets Enqueued during } \\
\text { Routing }\end{array}$ & 27600.00 & 11216.00 \\
\hline $\begin{array}{l}\text { The Total Packets Dequeued during } \\
\text { Routing }\end{array}$ & 27600.00 & 11216.00 \\
\hline The Total Packet Delivery Ratio & 100.00 & 615.67 \\
\hline
\end{tabular}

\section{CONCLUSION}

We have simulated a simplified model of GARUDA grid network in ns2. The normal data transfer protocol as well as the proposed content based data transfer protocol was simulated on the GARUDA grid network. As shown in the graphs in previous section, the use of content based data transfer scheme in application layer has obvious impact on network performance. The proposed scheme reduced the routing load very much since there were no duplicate flows in the network.

Future works may address the use of content based data transfer in other layers of Grid computing environment. More analysis can be made on the network with different operating conditions and cross traffic. Future works may address the possibilities of adding Quality of Services (QoS) aspects in the proposed content based data transfer scheme.

\section{REFERENCES}

[1] Bart Jacob, (June 2003),TSO Redbooks Project Leader, IBM,"Grid computing: What are the key components? - Taking advantage of Grid Computing for Application Enablement".

[2] Ann Chervenak, Ewa Deelman, Carl Kesselman,Bill Allcock, Ian Foster, Veronika Nefedova, Jason Lee, Alex Sim, Arie Shoshani,Bob Drach, Dean Williams,Don Middleton, (2001), "HighPerformance Remote Access to Climate Simulation Data: A Challenge Problem for Data Grid Technologies" - A technical document, Supercomputing Conference-SC.

[3] Antonio Carzaniga,Matthew J. Rutherford, Alexander L. Wolf, (2004), "A Routing Scheme for Content- Based Networking", Software Engineering Research Laboratory Department of Computer Science University of Colorado, Boulder, Colorado,USA, Technical Report CU-CS-953-03, June 2003 and IEEE INFOCOM.

[4] Baru, C., Moore, R., Rajasekar, A. and Wan, M., (1998), “The SDSC Storage Resource Broker. 8th Annual IBM Centers for Advanced Studies Conference”, Toronto, Canada.

[5] Beynon, M., Kurc, T., Catalyurek, U., Chang, C., Sussman, A. and Saltz, J. (2001) , "Distributed Processing of Very Large Datasets with DataCutter". Parallel Computing, 27 (11). 1457-1478. 
International Journal of Grid Computing \& Applications (IJGCA) Vol.2, No.4, December 2011

[6] Kurc, T., Catalyurek, U., Chang, C., Sussman, A. and Saltz, J. (2001), "Exploration and Visualization of Very Large Datasets with the Active Data Repository". IEEE Computer Graphics \& Applications, 21 (4). 24-33.

[7] Chervenak, A., Foster, I., Kesselman, C., Salisbury, C. and Tuecke, S, (2001), "The Data Grid: Towards an Architecture for the Distributed Management and Analysis of Large Scientific Data Sets", J. Network and Computer Applications (23). 187-200.

[8] Foster, I. and Kesselman, C. (2001), “A Data Grid Reference Architecture”, Technical Report GriPhyN-2001-12.

[9] Stockinger, H., Samar, A., Allcock, B., Foster, I., Holtman, K. and Tierney, B, (2002), "File and Object Replication in Data Grids". Journal of Cluster Computing, 5 (3). 305-314.

[10] Vazhkudai, S., Tuecke, S. and Foster, I., (2001), "Replica Selection in the Globus Data Grid. International Workshop on Data Models and Databases on Clusters And the Grid (DataGrid 2001)", IEEE Press.

[11] Foster, I., Kesselman, C., Tsudik, G. and Tuecke, S., (1998), "A Security Architecture for Computational Grids", 5th ACM Conference on Computer and Communications Security, 83-91.

[12] Hiroyuki Ohsaki, and Makoto Imase, (2009), "Performance Evaluation of Data Transfer Protocol GridFTP For Grid”, International Journal of Applied Mathematics And Computer Sciences Vol. 3, No.1,.

[13] Wen-Zhan Song and Xiang-Yang Li, (2004), “CBRBrain: Provide Content Based Routing Service over Internet Backbone”, In Proceedings of ICCCN'2004. pp.101-106

[14] Nader Mohameda,, Xin Liub, Amy Davisb, Byrav Ramamurthy, (2005) , "JOR: a content-based object router", Computer Communications $28,654-663$.

[15] Geoff Coulson, Paul Grace, Gordon Blair, Wei Cai, Chris Cooper, David, Duce Laurent Mathy, Wai Kit Yeung, Barry Porter, Musbah Sagar, Jason L, ( July 2006) ,"A Component-based Middleware Framework for Configurable and Reconfigurable Grid Computing”, Concurrency and Computation: Practice and Experience, Special Issue: First International Workshop on Emerging Technologies for Next- generation GRID (ETNGRID 2004), Vol.18. Issue 8, Pages 865-874.

[16] Piotr Grzybowski, Cezary Mazurek, Paweł Spychała, Marcin Wolski, Poznan Supercomputing and Networking Center, "Data Management System for Grid and portal services".

[17] Sasu Tarkoma,(2006)," Efficient Content-based Routing, Mobility-aware Topologies and Temporal Subspace Matching “, Report A-2, ISBN 952-10-3055-0.

[18] Zhen Li and Manish Parashar, (June 25-29, 2007) "A Computational Infrastructure for Grid-based Asynchronous Parallel Applications”, HPDC’07, ACM 978-1-59593-673-8/07/0006.

[19] Stefano Castelli, Paolo Costa,(2008), "HyperCBR: Large-Scale Content-Based Routing in a Multidimensional Space”, Conference: IEEE INFOCOM: 1714-1722.

[20] Roberto Baldoni, Roberto Beraldi, Leonardo Querzoni, Gianpaolo Cugola, Matteo Migliavacca, Politecnico di Milano, P.zza L. Da Vinci, (December 2005) , "Content-Based Routing in Highly Dynamic Mobile Ad Hoc Networks”, J. Pervasive Comput. \& Comm., Vol. 1 No. 4.

[21] Phillip M. Dickens, Illino, "A High Performance File Transfer Mechanism for Grid Computing" - A Technical Report, CoreGRID is a Network of Excellence funded by the European Commission the Sixth Framework Programme, Project no. 004265.

[22] Yi-Min Wang, Lili Qiu, Chad Verbowski, Dimitris Achlioptas, Gautam Das, and Paul Larson Microsoft Research, Redmond, (October 2004), "Summary-based Routing for Content-based Event Distribution Networks", ACM SIGCOMM Computer Communications Review 59 Volume 34, Number 5.

[23] Elke Michlmayr, Arno Pany,(2006), "Using Taxonomies for Content based Routing with Ants", A Funded project Report, under grant 31.963/46- VII/9/2002. Copyright is Held by the author/ owner(s).WWW2006, May 22-26, Edinburgh, UK. 
International Journal of Grid Computing \& Applications (IJGCA) Vol.2, No.4, December 2011

[24] Hari Subramoni, Ping Lai, Raj Ketti Muthu, Dhabaleswark (DK) Panda, (May 2010), "High Performance Data transfer in grid Environment using GridFTP over InfiniBand", Preceedings of the $10^{\text {th }}$ IEEE/ACM International Symposium on Cluster, Cloud and Grid Computing (CCGrid 2010).

[25] Wantao Liu, Brian Tieman, Rajkumar Ketti Muthu, Ian Foster, (2011), "Moving Huge Scientific datasets over the Internet", Pre-reviewed publications 2011.

[26] Thenmozhi. N, Madheswaran. M, (Sep 2010), "Analysis of Impact of Symmetric Encryption Algorithms in Data Security Model of Grid Networks”, IJCSIS, Vol 8, No.6, ISSN 1947-5500.

[27] http://partners.euindiagrid.eu/deliverables/D3.1.html

[28] http://www.eis.ernet.in

[29] www.garudaindia.in

[30] www.cdac.in

[31] www.griphyn.org

\begin{abstract}
Authors
Mrs. N. Thenmozhi is working as Assistant Professor, Department of Computer Science in N.K.R. Govt. Arts College for Women, Namakkal. She obtained her Bachelor degree in Statistics from Saradha College, Salem under Madras University, Master's degree in Computer Applications from Bharathiar University, Coimbatore, Master's degree in Software Systems from BITS, Pilani,and M.Phil From Manonmaniam Sundaranar University. She is currently pursuing Ph.D. under Mother Teresa Women's University, Kodaikanal. She has 18 years of Teaching Experience and 2 years of Industrial experience. She has published number papers in various national and international conferences. She is life member of ISTE. Her field of interest includes Grid Computing, Network Security and Image Processing.
\end{abstract}

M.Madheswaran received the BE Degree from Madurai Kamaraj University in 1990, ME Degree from Birla Institute of Technology, Mesra, Ranchi, India in 1992, both in Electronics and Communication Engineering. He obtained his $\mathrm{PhD}$ degree in Electronics Engineering from the Institute of Technology,Banaras Hindu University, Varanasi, India, in 1999. At present he is a Principal of Muthayammal Engineering College, Rasipuram, India. He has authored over Seventy five research publications in International and National Journals and Conferences. Currently he is the chairman of IEEE India Electron Devices Society Chapter. His areas of interest are theoretical modeling and simulation of high-speed semiconductor devices for integrated optoelectronics application, Bio-optics and Bio-signal Processing. He was awarded the Young Scientist Fellowship (YSF) by the State Council for Science and Technology,TamilNadu, in 1994 and Senior Research Fellowship (SRF) by the Council of Scientific and Industrial Research (CSIR), Government of India in 1996. Also he has received YSF from SERC, Department of Science and Technology, Govt. of India. He is named in Marquis Who's Who in Science and engineering in the year 2006. He is a Member of Institute of Electrical and Electronics Engineers, Fellow of Institution of Electronics and Telecommunication Engineers, Member of Indian Society for Technical Education and Member of Institution of Engineers. 